\title{
De la enseñanza de la bioética
}

a la educación bioética

Reflexiones sobre los desafíos

en los posgrados en Bioética

FROMTEACHING

BIOETHICSTO BIOETHICS

EDUCATION. REFLECTIONS

ONTHE CHALLENGES IN

THE DEGREE IN BIOETHICS

DO ENSINO DA BIOÉTICA

À EDUCAÇÃO BIOÉTICA.

REFLEXÕES SOBRE OS

DESAFIOS NOS PÔS-

GRAUS EM BIOÉTICA

$\checkmark 52$ Bioética

Jese Eirlos Abellán Salort*
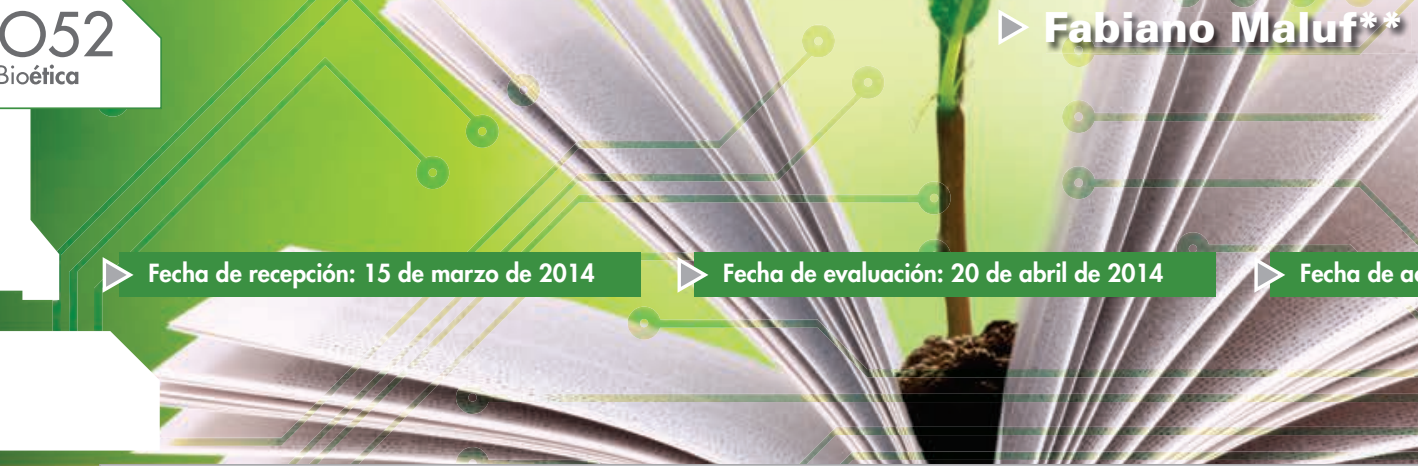

Doctor en Derecho. Profesor de Bioética y Derecho. Director del Máster Universitario en Bioética, de la Universidad Rey Juan Carlos, Madrid (España). Correo electrónico: jose.abellan@urjc.es

Odontólogo, magíster en Bioética e Investigador en Bioética. Cátedra Unesco de Bioética de la Universidad de Brasilia, Alumno de doctorado en Ciencias de la Salud en la Facultad de Ciencias de la Salud de la Universidad de Brasilia. Becario de CAPES - Proceso BEX nº 11519/13-4. Correo electrónico: 


\section{RESUMEN}

La importancia creciente de la reflexión Bioética ha fomentado la proliferación de programas de estudios específicos en esta materia, habitualmente centrados en modelos de enseñanza de carácter teórico, descriptivo, o bien en programas enfocados en análisis de casos prácticos, enseñanzas que, en última instancia, se corresponden con los modelos de argumentación y visiones dominantes de la Bioética. Desde un estudio comparado de los programas de posgrado (másteres universitarios) en Bioética que se ofrecen actualmente en España, los autores hacen un análisis sobre los principales desafíos para la enseñanza de la Bioética y defienden la necesidad de superar las limitaciones de los modelos de enseñanza (teóricos y casuísticos), a favor de programas de formación o educación integral que incluyan, en el perfil del formador, conocimientos suficientes de antropología y ética y que integren una visión amplia de lo que significa la vida y la dignidad de todo ser humano.

Palabras clave

Enseñanza de la bioética, educación en bioética, posgrado.

\section{ABSTRACT}

The growing importance of bioethics has encouraged the proliferation of specific programs of studies in this area, usually centered on theoretical teaching models or analysis of case studies, lessons that ultimately correspond to models of argumentation and dominant views of bioethics. From a comparative study of graduate programs (master's degrees) in bioethics currently offered in Spain, the authors make an analysis of the main challenges for teaching bioethics and defend the need to overcome the limitations of teaching models (theoretical and casuistic), in favor of comprehensive training or education, including on the teacher profile a sufficient knowledge of Anthropology and Ethics, and integrate a broad view of the meaning of life and dignity of every human being.

\section{Keywords}

Bioethics teaching, bioethics education, postgraduate.

\section{RESUMO}

A crescente importância da reflexão Bioética tem fomentado a proliferação de programas de estudos específicos nesta área, geralmente centrado nos modelos do ensino de caracter teórico, descritivo ou em programas focados no análise de casos práticos, ensinos que, em última instância, correspondem-se com os modelos de argumentação e visões dominantes de Bioética. A partir de um estudo comparativo dos programas de pós-graduação (mestrados universitários) em Bioética que hoje se oferecem na Espanha, os autores fazem uma análise dos principais desafios para o ensino de Bioética e defendem a necessidade de superar as limitações dos modelos de ensino (teóricos e casuísticos), em favor de programas para formação ou educação integral que incluam, no perfil do treinador, o conhecimento suficiente da antropologia e da ética e que integrem uma visão ampla do que significa a vida e a dignidade de todo ser humano.

\section{Palavras-chave}

Ensino da bioética, educação em bioética, pós-graduação.

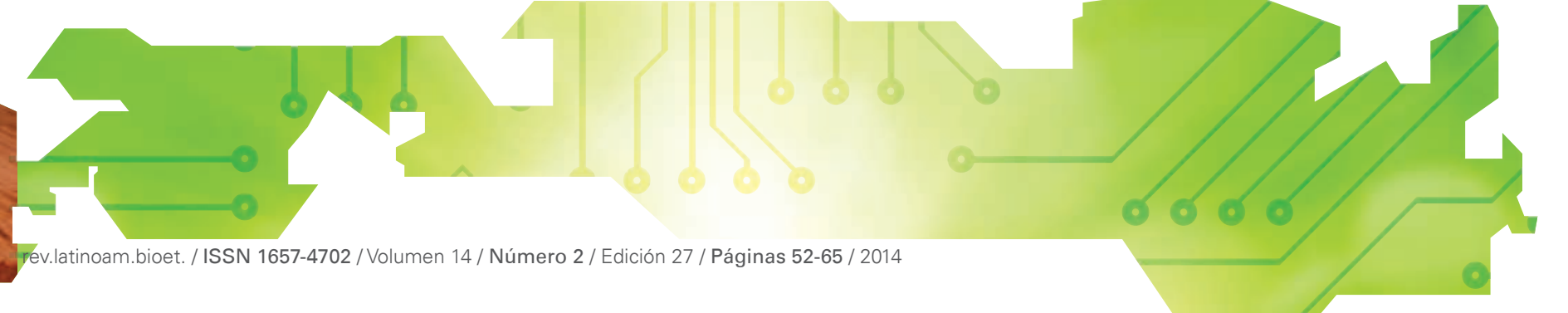




\section{INTRODUCCIÓN}

Con el acelerado progreso de la ciencia, sobre todo a partir de mediados del siglo XX, se implementó un modelo de enseñanza que favorecía la formación científica de alto nivel. Aunque los avances hayan contribuido al reconocimiento social de la ciencia médica y de otras profesiones del área de la salud, también contribuyó a la creación de una brecha entre la formación técnicacientífica y la formación ética y humanística.

Muchos autores critican que al énfasis que se da a este modelo de enseñanza tecnológico y científico no siguió la reflexión necesaria para la resolución de las cuestiones éticas y morales que surgieron con los avances en los conocimientos de biomedicina (Matos y Tenório, 2010; Silva y Ribeiro, 2009; Cezar, Gomes y Siqueira-Batista,

2011; Dantas, Martins y Militão, 2011; Caramico, Zaher y Rosito, 2007). Sin embargo, tener en cuenta también el contenido ético en la formación académica es cada vez más necesario. En este contexto, la enseñanza de la Bioética emergió como una posibilidad para la innovación curricular en los cursos de grado en Ciencias de la Salud como alternativa al modelo tecno-científico, prescriptivo y normativo.

Según Azevedo (1998), "al ser la Bioética un área compleja y reciente de conocimientos, no existe, para su enseñanza, una tradición pedagógica específica ni una experiencia didáctica consolidada" (p. 135). Entendida como disciplina científica o académica, la Bioética no se limita a la pedagogía tradicional, ni debería resumirse en una justificación pragmática basada en teorías preestablecidas.

Este estudio tiene como objetivo identificar, entre los másteres universitarios en Bioética ofrecidos en España, aquellos que tienen asignaturas de Antropología y Ética, hacer un análisis sobre los principales desafíos para la enseñanza de la Bioética, y se justifica con el propósito de superar las limitaciones de los modelos de enseñanza (teóricos y casuísticos), a favor de programas de formación o educación integral.

\section{SOBRE LA DIFERENCIA ENTRE ENSEÑANZA DE LA BIOÉTICA Y UNA FORMACIÓN O EDUCACIÓN BIOÉTICA}

Parece evidente la pertinencia, verdadera necesidad, de que la formación de los profesionales de las ciencias de la salud y de la investigación biomédica incorpore no solo una formación deontológica, sino también, complementaria con esta, una más amplia formación en Bioética. Pero, ¿qué fines, objetivos y alcance debería tener esa formación en Bioética? ¿Cómo habría de plantearse?

En este sentido, la formación en Bioética se viene estructurando, en los planes de estudio de las asignaturas de grado en los que se haya incorporado y también en los itinerarios formativos de posgrado (maestría y doctorado), de acuerdo con tres planteamientos distintos, alternativos, que creemos que responden a tres correspondientes enfoques de la misma ciencia Bioética.

1. Enfoque teórico-historicista-descriptivo

2. Enfoque casuístico

3. Enfoque humanístico-integral

Los dos primeros coincidirían con lo que podríamos denominar como enseñanza de la Bioética. El tercero se identificaría mejor con lo que defendemos como un necesario planteamiento de formación integral o educación Bioética.

Veamos en qué consiste cada uno de ellos.

Enfoque teórico-historicista-descriptivo. La formación en Bioética suele centrarse en una exposición descriptiva de la historia de la ciencia Bioética, habitualmente dentro de los estudios de deontología o de historia de la medicina, complementada con una explicación, más o menos completa de las corrientes ideológicas más representativas de la Bioética contemporánea (Postigo, 2007). Suele resumirse en una descripción panorámica de los principales documentos y aportaciones doctrinales de la Bioética occidental, a la que suele añadirse una referencia a los documentos del bioderecho internacional (declaraciones, recomendaciones de los organismos supranacionales y tratados internacionales) concernientes a los temas de la vida y la salud, y a la Bioética específicamente.

Se trata de una enseñanza (mostrar descriptivamente, empírica y descomprometida), de textos, documentos y doctrinas de la Bioética. No suele haber aplicaciones prácticas y generalmente se adolece de una pretensión de objetividad, en el sentido de no entrar a hacer valoraciones sobre los diversos modelos de argumentación expuestos. Este enfoque contiene elementos básicos de "cultura Bioética", y puede considerase en algún aspecto positivo y hasta necesario; además, cumpliría probablemente con los principales objetivos de una "enseñanza" de la Bioética, pero no es suficiente para una "educación" o formación integral en Bioética. 
Las razones fundamentales son:

1. No se proporciona al alumno herramienta intelectual alguna que le permita valorar, discriminar o jerarquizar entre los diversos modelos o doctrinas; la razón es que se renuncia, a priori, a proponer cualquier planteamiento metafísico o filosófico que pueda fundamentar un juicio.

2. No se aporta al alumno claves para juzgar los documentos bioéticos y biojurídicos, porque si no se cuenta con una formación antropológica, filosófica (ética) y jurídica básicas no se poseen criterios para valorarlos.

No se forma al alumno en habilidades para la decisión bioética, ya que no suelen realizarse actividades prácticas, ni se dota al alumno de competencias para resolver casos. Siendo así que la misión de la Bioética es proponer claves fundamentadas, que permitan al ciudadano, al profesional sanitario $y$ al legislador tomar decisiones éticamente responsables y correctas, un enfoque tan teórico no resulta adecuado.

Además, como consecuencia de hacer un enfoque que ha renunciado al juicio crítico o al análisis de las doctrinas y documentos, el resultado es una formación que presupone la validez de un cierto relativismo bioético. El estudiante, con este modelo academicista y descriptivo, termina su curso de Bioética con la impresión de que tiene igual validez un modelo que otro, y que en realidad su vigencia o mayor importancia responde a exigencias y necesidades históricas. Así mismo, algunos textos y corrientes son exaltados como si sus contenidos constituyeran dogmas irrefutables, sin advertir al alumno de sus limitaciones, sin presentarles las opiniones que pudieran cuestionar sus contenidos y, sobre todo, sin contextualizarlos adecuadamente.

Es legítimo optar por este modelo de enseñanza de la Bioética, pero advirtiendo al alumno que intencionalmente se renuncia al juicio, y consideramos engañoso y por tanto inaceptable presentarlo como absolutamente aséptico, porque esto último es sencillamente imposible y, por lo tanto, irreal.

3. Por último, renunciando a una formación más aplicada o casuística, parece pretencioso pensar que el alumno de un curso de Bioética así planteado sea capaz, solo por efecto de esta formación, de realizar el esfuerzo deductivo que implica la aplicación de lo estudiado a algo tan práctico como es la actividad de asesoramiento bioético, como tampoco que resulte habilitante, per se, para lo que se espera de un clínico o un investigador: que tome decisiones y que esas decisiones técnicas obedezcan también a un ponderado juicio bioético, prudente y equitativo, es decir, justo y en el caso concreto.

Enfoque casuístico. Este modelo de enseñanza de la Bioética parte de un objetivo fundamental: habituar al discente (profesional de la salud o de la investigación habitualmente) en la resolución de casos, en la deliberación Bioética, capacitarle para tomar decisiones en el ámbito clínico o investigador. Este enfoque posee, a primera vista, un valor indiscutible, en el sentido de que si uno de los fines esenciales de la Bioética es ayudar a conformar el recto juicio moral, a la luz de la razón, para la toma de decisiones concretas. Entonces, sin duda, conocer, analizar y debatir casos lo más cercanos a la realidad posible son una aportación positiva para la formación en Bioética.

Buena parte de los alumnos de cursos de Bioética tienen la aspiración de adquirir una formación que les habilite para ingresar en los comités asistenciales de ética hospitalarios (Abellán Salort, 2012), o bien

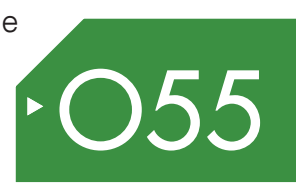
la cualificación académica que les faculte para promover a miembros de los comités de ética en investigación, órganos cada vez más presentes en las instituciones sanitarias y organismos y empresas de investigación biomédica y farmacéutica.

El asesoramiento bioético en este tipo de órganos se materializa mediante deliberaciones que se confía que culminarán en consensos que determinen recomendaciones de actuación concretas. Los consensos, habitualmente obtenidos por mecanismos de carácter democrático, presumen capacidades de análisis, competencias y conocimientos complementarias entre los miembros de los comités, intencionalmente representativos de las diversas áreas de interés de ese hospital, laboratorio o centro de investigación.

La naturaleza mayoritaria de las decisiones destiladas de las discusiones promovidas en estos órganos consultivos pretendería que se alcanza lo que mayoritariamente se entiende será la conducta mejor o menos mala, la 
Aducir razones como fundamento de estrategias o de decisiones bioéticas exige algo más que talante abierto y capacidad "negociadora"; hace indispensable conocer los "porqués" sobre la medicina, la investigación, en definitiva, sobre el ser humano. Exige tomar en consideración los fines, el telos médico-sanitario y el investigador, pero también hace ineludible fundar las razones en una jerarquía de bienes éticos que obligan a posicionarse sobre el valor y sentido de la salud y la vida humana. que parecerá más justa. Sin embargo, como en todo planteamiento ético basado en que lo justo es el resultado de un consenso pacífico mayoritario, por muy democrático que se garantice el procedimiento, la conclusión de la deliberación no necesariamente, no automáticamente y no siempre será lo verdadero o lo justo.

La apuesta por una enseñanza de la Bioética de carácter inductivo, casuístico, tiene una limitación de partida: el ser humano necesita razones, un fin o motivo, para decidir. Necesitamos argumentos racionales para inclinar nuestro juicio valorativo en un sentido o en otro, porque decidir, es jerarquizar. En el caso de la Bioética, las posiciones que se adoptan, de carácter ético, exigen discriminar entre bienes morales. La elección ética requiere de una fundamentación. $Y$ puestos a admitir que la deliberación pueda conducir a la resolución más cercana a lo bueno o a lo justo, nos parece poco creíble que el contenido del debate excluya "razones". Pues bien, los debatientes consultados, para exponer argumentos razonados, necesitan formación, no solo habilidades para el diálogo. Aducir razones como fundamento de estrategias o de decisiones bioéticas exige algo más que talante abierto y capacidad "negociadora"; hace indispensable conocer los "porqués" sobre la medicina, la investigación, en definitiva, sobre el ser humano. Exige tomar en consideración los fines, el telos médicosanitario y el investigador, pero también hace ineludible fundar las razones en una jerarquía de bienes éticos que obligan a posicionarse sobre el valor y sentido de la salud y la vida humana.

Por último, una enseñanza estrictamente casuística de la Bioética no puede presuponer que si el estudiante ha analizado, al término de la formación en Bioética, una serie de casos "de laboratorio" en el aula, ya posee las competencias para tomar decisiones fundamentadas cuando le lleguen casos parecidos en su vida real profesional. Porque no hay dos casos ni dos pacientes iguales, lo que exige consideraciones específicas en cada caso y situación, y porque las razones mayoritarias, o las estadísticamente más habituales, o las que adoptarían la opinión pública dominante, o las que le enseñaron como fruto de un consenso en el curso de Bioética no necesariamente son aplicables ni válidas en el siguiente caso real que se le presente.

El enfoque casuístico se conforma con una enseñanza práctica de la Bioética, que habitualmente supone renunciar a presentar al estudiante no solo la 
fundamentación necesaria, sino la misma verdad. La Bioética resultaría una actividad práctica para solucionar, mediante el consenso, conflictos éticos, desconectada de la posibilidad de la verdad y de alcanzar el bien, lo que ya supone un posicionamiento ideológico discutible.

Enfoque humanístico-integral. Los dos modelos anteriores son enfoques de la enseñanza de la Bioética, que poseen aspectos muy positivos expresados anteriormente, pero que presentan algunas deficiencias para lo que ambicionamos; debería ser una educación en Bioética, en el sentido de una formación y no solo de una enseñanza, pues implica una acción formativa más completa, más compleja y de mayor alcance. Más completa porque, junto a la descripción de la historia y de los diversos modelos de argumentación Bioética, debería incluir también el análisis de casos prácticos y poner al estudiante de Bioética ante situaciones verídicas o factibles de toma de decisiones en el ámbito clínicosanitario y en el de la investigación. Además, porque debería atender al tratamiento de asuntos de la ética del bios, (bio-ética es ética del bios, de la vida), pero de todo el bios, es decir, no solo de la vida humana, sino también del resto de vivientes, y de sus relaciones ecosistemáticas. Creemos que la Bioética debe incluir entre sus topics más importantes una ética ecológica y preguntarse por nuestros deberes morales en las conductas humanas que tienen como término al resto de los seres de la biosfera.

La Declaración Universal sobre Bioética y Derechos Humanos (DUBDH) (Unesco, 2005) ratifica nuestra posición en el Artículo 17 -Protección del medio ambiente, la biosfera y la biodiversidad- cuando afirma:

Se habrán de tener debidamente en cuenta la interconexión entre los seres humanos y las demás formas de vida, la importancia de un acceso apropiado a los recursos biológicos y genéticos y su utilización, el respeto del saber tradicional y el papel de los seres humanos en la protección del medio ambiente, la biosfera y la biodiversidad.

Finalmente, más completa porque en el desarrollo de una formación integral en Bioética es irrenunciable proponer al estudiante los diversos modelos de fundamentación de la Bioética. Por ello, creemos que resulta imprescindible formar al estudiante en las claves más relevantes de dos ciencias básicas para la Bioética: la antropología filosófica y la ética general.
En segundo lugar, la integración de todos estos elementos en el objeto material de estudio de la Bioética tiene como consecuencia una mayor complejidad de la enseñanza de la misma. La mayor complejidad de nuestro planteamiento formativo se debe a varios factores como:

1. Incorporar la capacitación para el razonamiento práctico en Bioética, con casos prácticos, debates, etc., exige una redefinición de las estrategias didácticas y las metodologías por parte de los profesores. En todas y cada una de las asignaturas - módulos del curso de Bioética, se debería combinar la acción formativa inductiva y la deductiva, de modo que se tengan aplicaciones prácticas en todas las sesiones de todas las materias o módulos, adaptándose al grado de conocimientos y madurez académica del grupo.

Esto significa estructurar los estudios de Bioética de modo que, progresivamente, se vayan incorporando elementos de casuística y aplicaciones prácticas, lo que debe hacerse con prudencia puesto que no puede haber una buena "práctica" si todavía no se ha interiorizado una buena "teoría".

La discusión de casos complejos no debería promoverse cuando el alumno todavía carece de los rudimentos intelectuales y la formación básica suficiente, pues la discusión no superaría el nivel emotivista o superficial de una conversación coloquial "de café". Por ejemplo, si se sitúa al alumno ante un caso de clonación humana sin los conocimientos básicos de biología celular y genética, o ante un caso de solicitud de retirada de un soporte vital o de eutanasia, sin haber profundizado en lo que implica

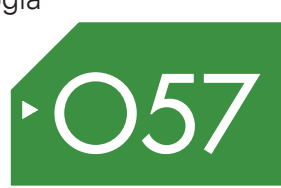
la libertad humana, o lo que es "muerte cerebral", por ejemplo.

La incorporación de ejemplificaciones, casos prácticos y debates sobre temas éticos y bioéticos desde el principio del programa, introducidos con prudencia, sin embargo tiene un efecto muy positivo sobre la motivación del alumnado, motivación que suele reforzarse con la conciencia de la utilidad práctica de sus estudios, para su vida profesional y personal.

2. La incorporación de la formación en fundamentos de antropología filosófica y ética general 
también hace más compleja la adquisición de los objetivos formativos. En primer lugar, porque nos encontramos con frecuentes prejuicios negativos hacia lo que representa una formación teórica, especialmente si esta tiene carácter filosófico, lo que hará conveniente a veces justificar ante el alumno la necesidad de estos estudios fundamentadores y explicando su utilidad para hacer una buena Bioética en el futuro. Además, será necesario familiarizarle con su lenguaje y metodología de estudio específicos.

Son relativamente frecuentes la incomprensión y hasta las reticencias de los alumnos cuando se encuentran con del hecho de que deben estudiar Antropología y/o Ética, no solo porque la mentalidad pragmática y antimetafísica de nuestra época favorece el rechazo a todo lo filosófico, sino también porque el alumno, procedente en su mayoría de estudios técnicos o biosanitarios, que dejó las humanidades de lado en su trayectoria de formación hace tiempo tiene que "esperar" a ver Bioética aplicada, o análisis de casos, y debe atravesar un territorio previo de formación filosófica, menos gratificante a priori y que quizá no esperaba encontrarse.

Una vez vencido el prejuicio, o primer rechazo si lo hubiere, los estudios de Bioética se hacen más complejos porque estas asignaturas o módulos requieren una metodología de estudio diferente al de la medicina, la genética o el derecho, en las cuales el alumno tiene que abrirse a lo filosófico, a lo metafísico, y a un modo de razonamiento práctico, el de la ética, para el que habitualmente no está familiarizado.

Será muy importante el acompañamiento tutorial de los alumnos en estas materias y también que los diversos profesores tengan la suficiente comunicación y coordinación entre ellos, de modo que en las clases y en los ejercicios prácticos se relacionen las diversas perspectivas. Por ejemplo, a la hora de explicar el concepto de muerte cerebral, será ineludible la referencia a los diversos conceptos o perspectivas de la "muerte" explicados en antropología; y a la hora de discutir un caso de riesgo de aborto voluntario terapéutico (el caso de tratamiento de gestante diagnosticada de cáncer, con riesgo claro para la salud del feto), serán de utilidad tanto las aclaraciones que se hicieron en Antropología sobre los conceptos de "vida humana", "persona", dignidad", etc., como también podrían serlo los principios de la ética general, explicados en ese módulo, como el principio ético de la "causa del doble efecto,", "mal menor", "totalidad", etc.

En cuanto al mayor alcance que se propone nuestra formación, si incorporamos estos elementos de carácter casuístico, y también los fundamentos de antropología y ética, es por una razón que se relaciona con la teleología de unos estudios de Bioética: ¿para qué nos formamos en Bioética? Si nos formamos con el único fin de agregar unos "barnices" humanísticos en nuestro currículum personal, o los cursamos para obtener una cualificación formal que nos permita integrar el comité ético del hospital, entonces probablemente nos bastaría con la enseñanza de la Bioética descriptiva o casuística que se suele ofrecer habitualmente.

\section{Una vez vencido el prejuicio, o primer rechazo si lo hubiere, los} estudios de Bioética se hacen más complejos porque estas asignaturas o módulos requieren una metodología de estudio diferente al de la medicina, la genética o el derecho, en las cuales el alumno tiene que abrirse a lo filosófico, a lo metafísico, y a un modo de razonamiento práctico, el de la ética, para el que habitualmente no está familiarizado. 
Sin embargo, lo que se propone aquí como una educación en Bioética es un esfuerzo más completo y más complejo, porque nos conecta con los fines de la actividad profesional y hasta con los fines últimos de toda vida humana. Un buen profesional, no solo del área de las ciencias de la salud, sabe que tiene el deber de realizar correctamente su tarea, su labor profesional diaria. A nadie se le escapa que este "buen hacer" profesional no se reduce al correcto desempeño técnico de su trabajo. Ser buen profesional exige el cumplimiento de deberes éticos y deontológicos.

Pero las normas de la ética profesional no se deberían cumplir "porque sí," o para evitar posibles sanciones del colectivo profesional previstas para el caso de incumplimiento. Un buen profesional cumple con esas reglas éticas porque sabe que ello le hace mejor profesional, porque realiza más plenamente los fines de esa profesión. Esto es lo que denominamos con el término griego telos. La satisfacción del telos de cualquier actividad nos remite a la puesta en acto de su esencia, a su realización perfectiva. Dicho coloquialmente, podríamos resumirlo en la idea de que, por ejemplo, un médico cumple plenamente con las exigencias que identifican esa profesión, cuando cumple con la ética profesional del médico, codificada en forma de deberes morales concretos, en el Código Deontológico.

La ética profesional, en el caso del médico, se justifica por los fines de la profesión médica: proveer la salud, curar y, cuando ello no es posible, cuidar y paliar el sufrimiento ajeno. Se orienta al bien del otro, en el caso del médico, al bien del paciente, comprendido como persona, digna y merecedora de ser tratada como tal. Cumpliendo sus deberes de respeto al paciente, a su intimidad, etc. deberes éticos, cumple más plenamente con los fines de su actividad y profesión sanitaria. Del mismo modo, para cualquier persona formarse en los aspectos éticos de la vida personal le ayudará a realizar acciones éticamente ordenadas, conforme a lo que son los fines de la vida humana.

Desde este último planteamiento descrito, la formación en Bioética quizá no debería situarse solo en el ámbito universitario, sino que debería adelantarse a las últimas etapas de la educación obligatoria secundaria y al bachillerato, cuando el adolescente y el joven se definen y reafirman en sus criterios éticos personales. Una correcta y completa formación en Bioética precisa de una buena formación en ética, en las bases generales del discurso ético, en los fundamentos de la decisión/acto moral y en los principios rectores del juicio moral. Requiere entender los procesos que la razón práctica humana realiza de forma habitual, sus fines, principios y elementos, esto es, dominar las bases de la ética general. Hay buenos manuales en español, para proporcionar esas bases al estudiante de Bioética que no tiene formación filosófica (Lucas, 2001).

Algunas de las limitaciones de algunos de los modelos de argumentación Bioética, y de los consiguientes modelos de enseñanza de la Bioética, tienen que ver con la pretensión de separar la Bioética de su ciencia "madre", que es la filosofía moral, pretendiendo construir una ética aplicada al margen, con plena independencia, de la ética que podríamos denominar "general".

Debe advertirse que cualquier teoría que construyamos sobre el acto bioético como acto moral, basándose en una correcta intelección del bien (ética), se fundamenta, en última instancia, en una antropología filosófica, es decir, en el conocimiento de que el hombre, con inteligencia racional y voluntad libre, es capaz de ese juicio prudente (Abellán Salort y Barahona, 2009).

Nuestra tesis es que la dinámica de la acción moral no es distinta en el ámbito de la valoración Bioética de las conductas humanas en el campo de las ciencias de la salud y de la vida; por el contrario, participa de los mismos presupuestos, pues la decisión Bioética es ética, moral. Es la decisión de un sujeto humano, capaz de lo moral (Drane, 2010). El estudiante y el estudioso de la Bioética deben conocer estos presupuestos epistemológicos y estrictamente antropológicos. No puede formarse en una ética aplicada a la investigación o a la práctica clínica si desconoce las bases antropológicas y filosóficas que hacen de una conducta buena y mala su contraria.

Incluso, dada la evidencia antropológica de la innata apertura del ser humano a la trascendencia y a lo religioso, y habida cuenta del relevante papel desempeñado por las religiones en el desarrollo de la Bioética occidental, sería importante completar la perspectiva filosófica, antropológica y jurídica, con el enfoque de la teología moral, y una panorámica suficiente de la comprensión del valor de la vida, la salud y la dignidad humanas, desde una perspectiva de religiones comparadas. 


\section{INVESTIGACIÓN SOBRE LOS CONTENIDOS DE ANTROPOLOGÍA Y ÉTICA EN LAS MAESTRÍAS UNIVERSITARIAS EN BIOÉTICA EN ESPAÑA}

Se trata de un estudio de carácter exploratorio descriptivo y este tópico tiene la finalidad de identificar todos los cursos de posgrado (maestrías universitarias) en Bioética ofrecidos en España, que tienen asignaturas con la denominación de Antropología y Ética.
Por medio de una consulta en la página web de las universidades seleccionadas, se localizó el contenido programático abordado durante todo el curso. Identificadas las asignaturas ofrecidas, se pasó al análisis de la existencia o no de disciplinas de Antropología y Ética o si estos contenidos eran contemplados en otras disciplinas. La tabla 1 muestra el registro de las disciplinas Antropología y Ética ofrecidas o no en las maestrías de Bioética en las universidades analizadas.

Tabla 1. Asignaturas de Antropología y Ética identificadas en las maestrías de Bioética en universidades y/o instituciones españolas

\begin{tabular}{|c|c|c|}
\hline & ANTROPOLOGÍA & ÉTICA \\
\hline 1. Cátedra Bioética Jèröme Lejeune & Sí & Sí \\
\hline 2. Fundación Universitaria Iberoamericana (Funiber) & Sí & Sí \\
\hline 3. Universidad Nacional de Educación a Distancia (UNED) & Sí & Sí \\
\hline 4. Universidad Católica San Antonio de Murcia (UCAM) & Sí & Sí \\
\hline 5. Universitat Ramon Llull + Institut Borja de Bioética & Sí & Sí \\
\hline 6. Universidad Rey Juan Carlos + Universidad San Pablo CEU & Sí & No \\
\hline 7. Universidad Católica de Ávila & Sí & No \\
\hline 8. Universidad Complutense de Madrid & No & Sí \\
\hline 9. Universidad Pontificia Comillas & No & Sí \\
\hline 10. Universidad de Navarra & No & No \\
\hline 11. Universidad de Murcia & No & No \\
\hline 12. Universidad Católica de Valencia & No & No \\
\hline
\end{tabular}

Fuente: elaboración propia.

Es oportuno hacer algunas consideraciones y observar particularidades sobre los resultados encontrados en el estudio durante la recolección de datos. La Cátedra de Bioética Jérome Lejeune, vinculada al Centro de Estudios Biosanitarios (CEB) (Madrid), ofrece su Máster en Bioética en la modalidad a distancia y su programa contempla una disciplina de Antropología y otra de Ética y moral, ambas con carga horaria de 40 horas y duración

de un mes. La Fundación Universitaria Iberoamericana

(Funiber) también ofrece su maestría en la modalidad a distancia y posee las disciplinas Bases antropológicas de la Bioética y Bases éticas de la Bioética, las dos con carga horaria de 30 horas.

Por su parte, la Universidad Nacional de Educación a Distancia (UNED) ofrece una maestría en Bioética en la modalidad a distancia y enel contenido programático se encontraron las disciplinas de Antropología y Bioética y "Fundamentos y métodos de la ética. La Universidad Católica San Antonio de Murcia (UCAM) ofrece su maestría de Bioética en la modalidad a distancia y los contenidos de antropología y ética se incluyen en las asignaturas Bases antropológicas de la Bioética y Fundamentos éticos de la Bioética, respectivamente, con tres créditos cada una.

La Universitat Ramon Llull en colaboración con el Institut Borja de Bioética en la ciudad de Barcelona ofrece en su maestría de Bioética, modalidad semipresencial, las asignaturas Antropología filosófica con 3,5 créditos e Introducción a la ética y a los diferentes sistemas éticos, con tres créditos. Algunas universidades, aunque no ofrezcan una disciplina exclusiva sobre antropología y ética, abordan estos contenidos en otras disciplinas. En la maestría de la Universidad Rey Juan Carlos, organizada en los últimos tres años con la colaboración con la Universidad San Pablo CEU, se ofrece la disciplina Antropología de seis créditos, como asignatura obligatoria, y el tratamiento de las cuestiones de ética general se contempla en la disciplina Fundamentos de Bioética. La maestría es ofrecida en la modalidad presencial. Está 
también la maestría en Bioética ofrecida en la modalidad semipresencial por la Universidad Católica de Ávila, la asignatura Fundamentos de Bioética, con 12 ECTS, está dividida en dos módulos: Antropología con seis ECTS y Moral fundamental, también con seis ECTS, pero no hay un módulo específico de ética.

En la Universidad Complutense de Madrid, actualmente no hay máster en Bioética, pero cuando se ofrecía no había una asignatura de antropología, pero sí un enfoque muy grande en la asignatura de Ética dividida en dos módulos presenciales: Ética fundamental que trabajaba los siguientes contenidos: Éticas teónomas, Éticas naturalistas, Éticas emotivistas, Éticas autónomas, Éticas utilitaristas, Liberalismo y ética, Éticas contratualistas y comunitaristas y Éticas de la responsabilidad; y Ética aplicada, en la cual se trata la ética de la relación clínica, la ética de la investigación clínica y biomédica, la ética y medicina intensiva, la ética ecológica.

En la Universidad Pontificia Comillas no hay asignatura de antropología en la maestría de Bioética, pero hay un abordaje antropológico, filosófico y teológico del dolor y la muerte en la asignatura Fundamentos de la ética del fin de la vida humana. También se identificó un amplio contenido relacionado con la ética en las asignaturas Fundamentos de la Bioética (tres ECTS, Ética, Moral y Bioética), Fundamentos filosóficos de la Bioética I-Filosofía clásica (tres ECTS, Ética aristotélica, Ética kantiana, Ética utilitarista, ética de los valores) y Fundamentos filosóficos de la Bioética II-Filosofía contemporánea (cuatro ECTS, (Ética dialógica, Ética de las virtudes y comunitarismo, Ética del cuidado, Ética de la responsabilidad de Hans Jonas).

En la maestría en Bioética ofrecida por la Universidad de Navarra, los contenidos de antropología se encontraron en el módulo La investigación en Bioética, con 3,75 ECTS, donde se trata la dignidad humana desde una perspectiva filosófica, y contenidos de ética en la asignatura La ética de las profesiones médicas, también con 3,75 ECTS, donde se propone un acercamiento a la ética cristiana y a la ética clásica. En la Universidad de Murcia, hay contenidos de antropología y ética en las competencias de las asignaturas Fundamentos y métodos de la Bioética (asignatura obligatoria de 12 ECTS) y Dilemas bioéticos en el origen de la vida humana (asignatura obligatoria de 12 ECTS), respectivamente.

Por último, la Universidad Católica de Valencia ofrece su maestría en la modalidad semipresencial y hay 
Dada la complejidad que implica la formación de los docentes en bioética, se espera de los profesionales que se dedican a la enseñanza de esta disciplina, comportamientos y prácticas que puedan servir de ejemplo para los estudiantes, con el fin de promover el desarrollo de una competencia política, social y esencialmente ética. contenidos de antropología filosófica y teorías éticas de Bioética, abordados en módulos en la asignatura obligatoria Fundamentos de Bioética, con seis ECTS.

\section{ALGUNOS DESAFÍOS EN LA ENSEÑANZA DE LA BIOÉTICA EN POSGRADO}

La Bioética se caracteriza por ser un área multi, inter y transdisciplinar (Garrafa, 2006) que está estrictamente relacionada con los problemas éticos que se desarrollan paralelamente a los avances científicos y tecnológicos de forma simultánea en diferentes lugares y culturas alrededor del mundo. Las características multi, inter y transdisciplinar, de manera didáctica, se pueden comprender de la siguiente manera (Nicolescu, 2000; Figueiredo, Garrafa y Portillo, 2008):

1. Multidisciplinariedad: se refiere al estudio de un objeto de una misma y única disciplina por varias disciplinas al mismo tiempo.

2. Interdisciplinariedad: se refiere a la transferencia de métodos de una disciplina para otra.

3. Transdisciplinariedad: se refiere a lo que está al mismo tiempo entre las disciplinas, es decir, entre los límites de sus diferentes fronteras, y tiene como uno de sus imperativos la unicidad del conocimiento.

Desde esta orientación epistemológica y didáctica, la estructura y organización de la enseñanza de Bioética deben adaptarse a la naturaleza de los contenidos, pero también al contexto sociocultural de la localidad donde se instale el curso, con el apoyo de marcos conceptuales y sobre la base de un conjunto de metodologías armónicas y científicas. Por ejemplo, mientras que "en los países de primer mundo, la enseñanza de la Bioética se orienta principalmente a los problemas éticos recurrentes de la aplicación de nuevas biotecnologías, en Sudamérica, los problemas bioéticos tienen una más grande amplitud" (Azevedo, 1998 pp. 128, 129). En este sentido, el enfoque debe estar dirigido a la diversidad de la salud social, económica, ambiental y pública.

Es importante destacar dos perspectivas de análisis de la Bioética desarrolladas en las diferentes culturas. La perspectiva angloamericana hegemónicamente principialista, cuya vital característica es la sobrevaloración del principio de autonomía (Abellán Salort, 2007), que es individualista y está fuertemente centrada en 
microproblemas (Azevedo, 1998). Y la perspectiva europea cuyo interés recae en la dimensión social del hombre, con atención a la moral colectiva (macroproblemas) y con un fuerte predominio del principio de la justicia (Matos y Tenório, 2010; Azevedo, 1998). Sin embargo, parece importante discutir determinados aspectos cuando se trata de la enseñanza de la Bioética; por ejemplo, ¿cómo enseñar y quién debe enseñar la Bioética? (Caramico, Zaher y Rosito, 2007; Azevedo, 1998; Zanatta y Boemer, 2007; Almeida et al., 2008).

En cuanto a la enseñanza de la Bioética, cabe considerar también qué metodología y recursos didácticos sean mejores para transmitir los contenidos a los alumnos. Promover debates es necesario dada la peculiaridad de la disciplina. Se trata de trabajar "con más argumentos y menos verdades" (Almeida et al., 2008, p. 441), y se vuelve difícil evaluar cuál es el mejor método de enseñanza "debido a la complejidad de medición de las actitudes y los comportamientos que, a menudo, implican valores y creencias" (Almeida et al., 2008, p.441).

Muchos métodos son utilizados por los profesores para la enseñanza de la Bioética; pueden citarse las clases teóricas (lección magistral), los seminarios y estudios de casos con dilemas bioéticos, las presentaciones en grupo con debates subsiguientes, los debates de textos y películas (cine foro) (Zanatta y Boemer, 2007), además de los simposios y conferencias simultaneas (Caramico, Zaher y Rosito, 2007).

Paralelo al dilema de las mejores dinámicas pedagógicas que se utilizarán por los profesores, está la cuestión de la carga horaria necesaria para el desarrollo de los temas bioéticos. El número ideal de horas es dependiente y está estrechamente relacionado con la cantidad de tiempo necesario para desarrollar apropiadamente el contenido (Odom, 1988). En este sentido, una carga horaria insuficiente en la disciplina no permite trabajar los contenidos correctamente, hecho que imposibilita a los estudiantes la maduración frente a las posturas éticas necesarias, antes los conflictos y dilemas que enfrentarán en la vida profesional (Camargo, 1996).

Figueiredo (2011) encontró que la enseñanza de la Bioética en los estudios de posgrado stricto sensu, en el área de ciencias de la salud, oscila entre 10 a 20 horas, 20 a 30 horas y 40 a 50 horas, que la carga horaria más frecuente es de 25 horas y que ocurre en 79 de las disciplinas analizadas. Resultados similares fueron encontrados por Aires et al. (2006), que informaron que el número promedio de horas dedicadas a la enseñanza de la Bioética en los programas de posgrado en Odontología en Brasil es de 36 horas lectivas. Según Kottow (2009), no hay una carga horaria optima prestablecida y consensuada. La Bioética suele ocupar generalmente los espacios que consigue ganar en la estructura curricular.

Se defiende en hecho de que trabajar en pequeños grupos suele ser la más adecuada forma para la formación en Bioética (Zanatta y Boemer, 2007; Dantas y Sousa, 2008; Finkler et al., 2011), donde se estimula, de manera abierta y democrática, la confrontación de ideas y creencias (Dantas y Sousa, 2008). Por lo tanto, la combinación de clases teóricas con actividades prácticas-seminarios, defendidos y analizados en pequeños grupos, parece ser la estrategia preferida de los estudiantes y más a menudo adoptada por docentes (Kottow, 2009), aspecto que favorece el encuentro entre los actores del proceso-profesor y alumno (Finkler et al., 2011).

Dada la complejidad que implica la formación de los docentes en Bioética, se espera de los profesionales que se dedican a la enseñanza de esta disciplina comportamientos y prácticas que puedan servir de ejemplo para los estudiantes, con el fin de promover el desarrollo de una competencia política, social y esencialmente ética (Mascarenhas y Santa-Rosa, 2010). Figueiredo (2011b), al estudiar el perfil de los profesores de Bioética en una institución que ofrece posgrado en Brasil, verificó que el $86,47 \%$ de los profesores analizados mediante un estudio de sus currículos no tienen formación específica en Bioética, sea a nivel de especialización, de maestría o doctorado.

A partir de los datos encontrados por el autor, nos preguntamos qué perfil debería tener un profesor de Bioética, qué formación académica y experiencia debería reunir, y cuáles son los profesionales más adecuados para enseñar una disciplina tan amplia y compleja (Figueiredo, 2011b).

\section{EL PERFIL DEL FORMADOR EN BIOÉTICA}

Una de las cuestiones más controvertidas a la hora de programar la educación o formación en Bioética es la determinación de las características o perfil del profesor. En este punto, creemos importante considerar algunas cuestiones previas: 
1. La naturaleza esencialmente interdisciplinar de la ciencia Bioética, en la que confluyen datos y contenidos procedentes de las ciencias empíriconaturales (biología, genética, etc.), junto con la filosofía (ética, antropología, etc.) y las ciencias jurídicas (biojurídica, bioderecho aplicado, nacional e internacional). Este carácter interdisciplinar de la Bioética exigiría, aparentemente, formadores versados en estas ciencias o incluso en varias de ellas simultáneamente, lo que es complicado de encontrar.

2. La juventud de la Bioética, como ciencia y como disciplina académica, con solo cuarenta años de historia, lo que explica que, hasta el inicio de este siglo no existieran apenas cátedras o centros de investigación especializados ni demasiados programas de formación especializados (Drane, 2010).

Nuestra experiencia en diversos programas y al contraste con las opiniones de otros formadores en Bioética es que un buen profesor de un programa de Bioética debería ser, ante todo, un profesional docente cualificado, especialista en al menos una de las tres grandes áreas señaladas anteriormente. Además de lo anterior (ser un buen biólogo, o genetista, o filósofo o jurista, etc.), el formador en Bioética debería poseer también conocimientos siquiera básicos de biología humana, antropología y ética o derecho, y además formación específica en Bioética, sea porque ha estudiado un Grado Universitario, o porque posee un título de maestría o curso experto en Bioética.

La razón es que, para afrontar con éxito la formación en Bioética, resulta ineludible que el docente haya realizado antes esa "síntesis de saberes", esa conexión interdisciplinar que exige un estudio adecuado de los topics de la Bioética. Esto no significa que, para explicar Bioética, además de ser médicos, por ejemplo, haya que tener la carrera de Filosofía o saber mucho acerca del derecho. Pero seguramente sí lo será poseer conocimientos básicos de estas otras disciplinas (por ejemplo, no siendo médico, conocer mínimamente lo que es una célula, o una proteína, o qué significa antropológica y jurídicamente el término "persona", etc.).

El formador en Bioética, poseedor de esa formación interdisciplinar, debería proponerse el reto de provocar la reflexión rigurosa, sobre los conflictos y casos de la Bioética, en el alumno, y al mismo tiempo proponerle claves y criterios metabioéticos que puedan fundamentar ese juicio bioético. Por otra parte, debe ser capaz de motivar al alumno para que realice esa conexión o síntesis de saberes, promover la búsqueda honesta de la verdad y del bien, y asegurar una sólida formación metaBioética del discente que le faculte para evaluar críticamente las situaciones Bioéticamente relevantes.

Por último, es importante que el docente de Bioética sea capaz de establecer las bases y enseñar las diversas metodologías de deliberación y argumentación Bioética, así como las claves para investigar en Bioética, puesto que la investigación en materias de carácter humanístico como la Bioética tiene sus propias fuentes, metodología y caracterización diferenciales.

\section{CONSIDERACIONES FINALES}

Un planteamiento adecuado de las prácticas investigadora, clínica y asistencial debería estimular la búsqueda de nuevas formas de abordar la realidad, que no puede ignorar la reflexión ética (Pires y Garrafa, 2011). Por lo tanto, la formación en Bioética a través de cursos de posgrado, debe entenderse como parte de un proceso de construcción de la práctica profesional, a partir de la mejora de la dimensión ética y del desarrollo de valores y compromisos con bases más sólidas, más allá del deseo y la intuición (Matos y Tenório, 2010).

En relación con la definición de los objetivos de la enseñanza de Bioética en el posgrado, el primer desafío sería analizar cómo transmitir el conocimiento desde una visión interdisciplinar, fomentando la reflexión crítica sobre las actitudes, bienes y valores que concurren en los asuntos bioéticos (Correa, 2008). En una disciplina tan amplia y compleja, establecer metas predeterminadas sería una actitud poco consistente como para determinar qué temas deben formar el plan de estudios. En este sentido, se convierte en un reto elaborar un menú para la enseñanza de la Bioética que, atendiendo la multiplicidad de disciplinas involucradas, muestre la creciente incorporación de las nuevas tecnologías con implicaciones morales en la vida cotidiana de las personas (Caramico, Zaher y Rosito, 2007).

En conclusión, si apostamos por una Bioética que responda a las necesidades de la sociedad y pueda ayudar a sanitarios, biotecnólogos, investigadores, políticos y 
jueces a conformar decisiones y actuaciones correctas y justas, necesitamos contar con auténticos bioeticistas; esto es, expertos en Bioética formados integralmente, conocedores del bios y del ethos; de la persona, y del ius; es decir, capacitados para afrontar los dilemas bioéticos con la solvencia de quien conoce la verdad sobre el ser humano, su vida y su dignidad. Ello implica ampliar la perspectiva de la enseñanza de la Bioética, hacia una educación o formación integral en Bioética, con una sólida fundamentación en antropología y ética.

\section{REFERENCIAS}

-

Abellán Salort, J.C. (2012). Los CAES: Comités Asistenciales de Ética en la Atención Primaria en España. Un trabajo diferente. En AA.VV.: Bioética, poderes y justicia. Diez años después. Consejo Federal de Medicina, Cátedra Unesco de Bioética. Sociedade Brasileira de Bioética. Brasília.

- Abellán Salort, J.C. (2007). Bioética, autonomía y libertad. Madrid: Universitaria Española.

- Abellán Salort, J.C. y Barahona, M.L. (2009). Los Códigos de la Vida. Madrid: Homo Legens.

- Aires, C.P.; Hugo, F.N.; Rosalen, P.L. y Marcondes, F.K. (2006). Teaching of bioethics in dental graduate programs in Brazil. Brazilian Oral Research, 20(4), 285-289.

- Caramico, H.J.; Zaher, V.L. y Rosito, M.M.B. (2007). Ensino da Bioética nas faculdades de medicina do Brasil. Bioethikos, 1(1), 76-90.

- Cezar, P.H.N.; Gomes, A.P. y Siqueira-Batista, R. (2011). O cinema e a educação Bioética no curso graduação em medicina. Revista Brasileira de Educação Médica, 35(1), 93-101.

- Dantas, A.A.; Martins, C.H. y Militão, M.S.R. (2011). O cinema como instrumento didático para a abordagem de problemas bioéticos: uma reflexão sobre a eutanásia. Revista Brasileira de Educação Médica, 35(1), 69-76.

- Dantas, F. y Sousa, E.G. (2008). Ensino da deontologia, ética médica e Bioética nas escolas médicas brasileiras: uma revisão sistemática. Revista Brasileira de Educação Médica, 32(4):507-17.

- De Moura Almeida, A.; Vieira Bitencourt, A.G..; Branco Cequeira Neves, N.M.; Branco Cequeira Serra Neves, F.; Da Rocha Lordelo, M.; Moreira Lemos, M.; Ribeiro Nuñez, G.; Campos Barbetta, M.; Abenzur Athanazio, R. y Nery-Filho, A. (2008). Conhecimento e interesse em ética médica e Bioética na graduação médica. Revista Brasileira de Educação Médica, 32(4), 437-44

- De Sousa E Azevedo, E.E. (1998). Ensino de Bioética: um desafio transdisciplinar. Interface, 2(2), 127-37.

- Drane, J.F. (2010). La disciplina de la Bioética en España y en las Américas. Una historia personal. En B. Herreros y F. Bandrés (eds.), Educar en Bioética al profesional de ciencias de la salud. Una perspectiva internacional. Madrid.

- Figueiredo, A.M. (2011a). O ensino da Bioética na pós-graduação stricto sensu, na área de Ciências da Saúde, no Brasil. Revista Brasileira Pós Graduação, 8(15), 139-61.

- Figueiredo, A.M. (2011b). Perfil acadêmico dos professores de Bioética nos cursos de pós-graduação no Brasil. Revista Brasileira de Educação Médica, 35(2), $163-70$.
Figueiredo, A.M.; Garrafa, V. y Portillo, J.A.C. (2008). Ensino da Bioética na área das ciências da saúde no Brasil: estudo de revisão sistemática. Revista Internacional Interdisciplinar INTERthesis, 5(2), 47-72.

- Finkler, M.; Verdi, M.I.M.; Caetano, J.C. y Ramos, F.R.S. (2011). Formação profissional ética: um compromisso a partir das diretrizes curriculares? Trabalho, Educação e Saúde, 8(3), 449-62.

- Garrafa, V. (2006). Multi-inter-transdisciplinaridade, complexidade e totalidade concreta em Bioética. En V. Garrafa, M. Kottow y A. Saada A. (orgs.), Bases conceituais da Bioética, enfoque latino-americano (pp.7385). São Paulo: Editora Gaia.

- Kottow, M.H. (2009). Enseñanza de la Bioética: una síntesis. Revista Brasileira de Educação Médica, 33(4), 658-63.

- León Correa, F.J. (2008). Enseñar Bioética: como transmitir conocimientos, actitudes y valores. Acta Bioethica, 14(1), 11-7.

- Lucas, R. (2001). Antropología y problemas bioéticos. Madrid: BAC.

- Mascarenhas, N.B. y Santa-Rosa, D.O. (2010). Bioética e formação do enfermeiro: uma interface necessária. Texto \& Contexto Enfermagen, 19(2), 366-71.

- Matos, M.S. y Tenório, R. (2010). Percepção de alunos, professores e usuários acerca da dimensão ética na formação de graduandos de odontologia. Ciência \& Saude Coletiva, 15(supl. 2), 3255-64.

- Nicolescu, B. (org.) (2000). Um novo tipo de conhecimento: transdisciplinaridade. En Educação e transdisciplinaridade (p. 10). Brasília: Unesco.

- Odom, J.G. (1988). The status of dental ethics instruction. Journal Dental Education, 52(6), 306-8.

- Pires, J. y Garrafa, V. (2011). Educação: nova fronteira da Bioética. Ciência \& Saude Coletiva, 16(supl. 1), 735-45.

- Postigo, E. (2007). Bioética, antropología y corrientes éticas. En G.M. Tomás y Garrido y E. Postigo Solana (eds.). Bioética personalista: ciencia y controversia. Madrid: Eiunsa.

- Silva, R.P. y Ribeiro, V.M.B. (2009). Inovação curricular nos cursos de graduação em medicina: o ensino da Bioética como uma possibilidade. Revista Brasileira de Educação Médica, 33(1), 134-43.

- UNESCO (2005). Declaração Universal sobre Bioética e Direitos Humanos. Recuperado de http://www.sbbioetica.org.br/wpcontent/uploads/2011/11/ TEXTODADUBDH.pdf

- Von Zuben de Arruda Camargo, M.C. (1996). O ensino da ética médica e o horizonte da Bioética. Bioét, 4(1), 47-51.

- Zanatta, J.M. y Boemer, M.R. (2007). Bioética: uma análise sobre sua inserção nos cursos de graduação em enfermagem em uma região do Estado de São Paulo. Bioethikos, 1(2), 63-9. 